\title{
La educación en el ciberespacio; Una prospectiva a corto plazo de las clases a distancia de los alumnos de posgrado.
}

\author{
Ciberspace academic; A short-term foresight of distance classes for \\ graduate students.
}

\begin{abstract}
Sánchez Reséndiz, Christopher ${ }^{1}$
${ }^{1}$ Instituto Politécnico Nacional, Escuela Superior de Ingeniería y Arquitectura Unidad Tecamachalco, Sección de Estudios de Posgrado e Investigacion, Doctorado en Ciencias en Arquitectura y Urbanismo. Naucalpan de Juárez; Estado de México. México. 1csanchezr1008@alumno.ipn.mx / https://orcid.org/0000-0002-9426-2313
\end{abstract}

Recibido el 08 de abril de 2021, aprobado el 03 de junio de 2021

RESUMEN | El ciberespacio, se ha establecido como el principal espacio en el último año, motivo por la contingencia del COVID-19, asi mismo, esta dinámica ha venido a establecer algunos paradigmas para comprender "la nueva normalidad", tales como, la educación a distancia, de este modo, los alumnos e investigadores de la Sección de Estudios de Posgrado e Investigación (SEPI) de la Escuela Superior de Ingeniería y Arquitectura (ESIA) Unidad Tecamachalco del Instituto Politécnico Nacional (IPN), por motivos salubres, se han visto obligados a interactuar $\mathrm{y}$ adecuarse ante las herramientas tecnológicas y digitales disponibles.

El siguiente ejercicio hace referencia a la educación a distancia y los escenarios hipotéticos del ciberespacio en torno a los estudiantes de posgrado, así mismo, se da un panorama en la concepción de lo que es el ciberespacio y su relación con el espacio tangible, por lo que, abre a discusión, ¿Hacia dónde se dirige la enseñanza de la arquitectura y urbanismo?, ¿Cuáles serían los futuros retos para el discente y docente?, ¿Qué estrategias ayudarían a comprender y percibir un espacio urbano arquitectónico por medio de las herramientas electrónicas y digitales?, ¿la enseñanza de la arquitectura junto con el urbanismo, tendrán la misma visión como se ha venido dando antes de la pandemia?, y, ¿Qué prospectiva tendrá la enseñanza de la disciplina para generar interés asi como el beneficio de la sociedad?, por lo que para responder estas preguntas se requirió a entrevistas semiestructuradas.

PALABRAS CLAVE | Ciberespacio, prospectiva, educación, arquitectura, urbanismo

ABSTRACT | Cyberspace, it has established itself as the main space in the last year, reason for the contingency of COVID-19, likewise, this dynamic has been established to establish some paradigms to understand "the new normality", such as distance education, in this way, students and researchers of the Posgraduate and Researching Study Section (PRSS) from Engineering Architecture Superior School (EASS) Tecamachalco Unity from Nacional Institute 
Polythecnic (NIP), for health reasons, have been forced to interact and adapt to the technological and digital tools available.

The following exercise refers to distance learning and the hypothetical scenarios of cyberspace around graduate students, as well as a picture of the conception of what cyberspace is and its relationship with tangible space, so it opens up for discussion, where is the teaching of architecture and urbanism headed? , What would be the future challenges for the students and teachers?, What strategies would help to understand and perceive an architectural urban space through electronic and digital tools?, the teaching of architecture together with urbanism, will have the same vision as has been given before the pandemic?, and, what foresight will the teaching of discipline have to generate interest as well as the benefit of society?, so to answer these questions, semi structured interviews were required.

KEYWORD | Cyberspace, foresight, education, architecture, urban planning.

\section{Introducción}

El presente trabajo retoma lineamientos teóricos y empíricos implementando la metodología de entrevistas semiestructuradas realizadas a 10 compañeros de la SEPI ESIA Tecamachalco, además de referentes estadísticos que aportan una perspectiva más que nos permitirá conocer el fenómeno a prospectivar desde una visión compuesta por el mercado, la academia y los puntos de vista subjetivos de quienes se han adaptado a la nueva normalidad. Tratar temas como el de ciberespacio y sus implicaciones a nivel urbano-arquitectónico resulta urgente en contextos que se pensaban muy poco probables hace unos meses, con lo cual se confirma la urgencia de plantear escenarios de futuro que nos permitan reaccionar de mejor manera al impedir tragedias como la que estamos viviendo.

El ciberespacio se ha venido consolidando a lo largo de varias décadas, desde que los medios de comunicación que no involucran a los sujetos en un mismo espacio hicieron su aparición, sin embargo, el avance en las tecnologías de la información y su papel cada vez más relevante en la economía global forma parte de un acceso cada vez mayor a dispositivos electrónicos como son computadoras y teléfonos celulares, de modo que lo exclusivo y poco accesible se ha convertido en el medio más común a nivel mundial para compartir y generar información, sin olvidar que hay poblaciones enteras sin acceso primero a electricidad y por consecuencia el internet, de manera que al mismo tiempo, se trata de herramientas excluyentes.

Lo anterior y con las limitaciones que supone, nos lleva a un momento paradigmático que inició hace un año, en México, las interacciones cara a cara fueron paulatina o bruscamente reemplazadas por la intermediación de una pantalla, la contingencia ocasionada por el COVID-19, fue el detonante de lo que ya se venía utilizando como medio alternativo, es decir, que reemplazaba la presencia física de ser necesario.

Lo que se ha dado por nombrar como "nueva normalidad" impone restricciones a las relaciones interpersonales, la utilización de cubrebocas, caretas, guantes, gel anti bacterial y una serie de medidas de distanciamiento que restringen la actividad cotidiana y donde las interacciones virtuales encuentran su máxima expresión, por ser éstas las más seguras, tanto para realizar compras, como para trabajar o conversar y saber de los seres queridos. 
Nuestro interés se centra en la educación, uno de los tópicos más impactados por las condiciones contemporáneas de riesgo, debido, entre otros motivos a las aglomeraciones que suponen los salones de clases, pasillos y demás entornos escolares. Para el presente texto retomamos el caso de los alumnos e investigadores de la SEPI, de la ESIA Tecamachalco del IPN, quienes, de manera análoga al resto de estudiantes del país y siguiendo los protocolos de salubridad, interactúan por medio de herramientas tecnológicas y digitales.

A partir de lo anterior, nos centramos en la educación telemática, por lo que el objetivo es exponer y plantear escenarios hipotéticos de un futuro a corto plazo, en el que los estudiantes del posgrado se plantean la relación existente entre el ciberespacio y el espacio tangible o arquitectónico, de manera que los límites entre habitar, trabajar, estudiar, vida familiar y académica son cada vez menos precisos y sin duda, es necesario preguntarse por un futuro en el que la convivencia entre dichos ámbitos estará cada vez más naturalizada, formando parte de la cotidianidad con o sin pandemia.

\section{De la prospectiva al Ciberespacio, concepciones teóricas y estadísticas para explicar la educación telemática.}

Plantear escenarios futuros requiere de conocer el fenómeno a prospectivar, para lo cual recurrimos a las tres de las etapas de Gandara (2014) como método; en primer lugar con el "entendimiento del contexto"; que nos permita identificar las variables y la comprensión del pasado, además del aspecto cuantitativo, para el cual se retoman las estadísticas, sin perder de vista nuestro concepto guía que es el de "ciberespacio", en segundo lugar, "la visualización estratégica" nos permite categorizar las variables que se relacionan con los actores, es decir, con los estudiantes de la SEPI, quienes forman parte de un tiempo-espacio sin precedentes históricos que plantea múltiples emociones, sensaciones corporales y formas distintas de aprender, además de un futuro que plantea escenarios poco claros o inciertos.

Por último, realizaremos el ejercicio fundamentado de "dibujar los futuros" al plantear los posibles escenarios hipotéticos, por medio de los referentes teóricos en correlación con la información de los actores, llegando a la "construcción de futuros" donde se seleccionan los escenarios tendenciales, futuribles, utópicos y pesimistas, ya que dentro de esta etapa final se relacionan los intereses y los desafíos que implica concebirlos.

\section{Pandemia y ciberespacio, contexto histórico para la SEPI.}

A inicios del año 2020, la cotidianidad para los alumnos de la SEPI de la ESIA Tecamachalco, marchaba como siempre, es decir que, consistía en asistir a las aulas de clases; en la Unidad Tecamachalco y/o en la Unidad Regina, esta última ubicada en el primer cuadro del Centro Histórico de la Ciudad de México, además de participar en congresos, coloquios, trabajo de campo, visitas a lugares importantes y revisiones de avances de tesis, por no mencionar las actividades relacionadas con la asistencia a la escuela, como trasladarse, comer, tomar un café, conversar y generar vínculos afectivos con los compañeros.

Ya para mediados del mes de marzo del 2020, llegamos al inicio de una visión un tanto híbrida y hasta hace poco futurista, que desdibujaba la interrelación entre espacio arquitectónico y espacio virtual, lo que anteriormente sólo se mencionaba en visiones como la de Doxiadis (1974) la Ecumenópolis y Mitchell (2000) con E-topia, más cercana a los dispositivos digitales que hoy se conocen. 
Se podrían seguir relacionando de una forma tradicional los espacios inteligentes servidos y de servicio, haciendo que sean adyacentes, pero también se podría establecer su conexión funcional a través de un remoto enlace electrónico. la organización funcional del espacio arquitectónico se puede seguir deduciendo de las plantas y de los mapas de uso del suelo, pero ahora también se debe tener en cuenta el componente de las redes y los programas (Mitchell, 2000, p.135)

Además, Mitchell (2000) mencionaba algunos escenarios urbanos, similares a los que estamos presenciando, es decir, el ciberespacio como forma de vida cotidiana. Asimismo, en el transcurso de los meses que nos traen hasta ahora, enero del 2021, la sociedad en su conjunto ha tenido que adaptarse por obligación a esta "nueva normalidad"; la pandemia de Covid-19 ha expuesto las carencias, las debilidades, las desigualdades y la constante necesidad de adaptación tanto en México como en el mundo.

La denominada, "nueva normalidad" plantea lineamientos técnicos y protocolos de seguridad sanitaria implementados por la Secretaría de Salud en México, éstos dependen del color del semáforo de riesgo epidémico en cada entidad del país, con la siguiente categorización:

Verde: se permiten todas las actividades, incluyendo las escolares.

Amarillo: hay actividades, pero con precaución, todas las actividades económicas están permitidas con medidas de cuidando a las personas de mayor riesgo.

Naranja: quedarse en casa en la medida de lo posible, con las actividades económicas no esenciales a un $30 \%$ de funcionamiento.

Rojo: no salir a menos que sea estrictamente necesario, con actividades económicas esenciales.

Lo anterior ha generado polémicas distintas en los ámbitos de economía, salud, religión y educación principalmente. En el ámbito económico los comerciantes de giros denominados como no esenciales demandan abrir sus locales para reactivar las ventas, por su parte, los hospitales presentan una saturación sin precedentes, mientras para el caso de la educación, el regreso a las actividades presenciales se pospone mes con mes, de manera que el tema de la educación telemática ha tenido ventajas y desventajas que analizaremos con mayor profundidad en el apartado de las entrevistas, puesto que los alumnos de la SEPI de la ESIA Tecamachalco han tenido que acostumbrarse a esta nueva dinámica.

La utilización de nuevas tecnologías forma parte del Modelo Educativo 4.0, que se basa en la comunicación como principal elemento, con ello, la colaboración entre alumnos y docentes se vuelve más horizontal, con el juego como uno de los principales elementos de enseñanza (Game Based Learnign GBL), y las TIC's como elementos imprescindibles. Dicho modelo se ha comenzado a implementar en los planes de estudio del IPN:

La educación 4.0 tiene un papel importante en el Politécnico, porqué el Instituto siempre ha respondido a las necesidades del desarrollo nacional. Una de sus características es que más de un millón de egresados está contribuyendo en el desarrollo del comercio, de la producción, de la construcción, de los servicios en el sector social. Por eso es que la sociedad reconoce al IPN por el impacto que ha tenido en el desarrollo del país y en la calidad de vida de los ciudadanos mexicanos (Toro, 2019)

Lo anterior se confirma con el hecho de que, incluso antes de la contingencia sanitaria, el IPN 
contemplaba algunos programas académicos no presenciales y mixtos:

Tabla 1: Programas académicos ofertados por el IPN. Fuente: Elaborado por el autor.

\begin{tabular}{ccc}
\hline Programas académicos & Modalidades & Total, de programas académicos \\
\hline Licenciatura & No Escolarizado & 8 \\
\hline Licenciatura & Mixto & 1 \\
\hline Posgrado & No Escolarizado & 6 \\
\hline
\end{tabular}

Llegar a lugares alejados brindando la educación con el nivel que se requiere para formar profesionales es posible a partir de los medios de comunicación, “Aquí la educación virtual juega un papel fundamental, el poder desarrollar unidades de aprendizaje a distancia, que sean masivas, respondan a la necesidad" (Toro, 2019), de manera que la educación sea accesible para acortar la brecha entre grandes ciudades y comunidades rurales.

Este contexto plantea la posibilidad de generar diversas reflexiones que nos permitan llegar a una visión de corto plazo, de inicio, cabe preguntarse si la SEPI de la ESIA Tecamachalco; ¿Se encuentra lista para una Educación Telemática?, en consecuencia, ¿Cuál sería el futuro de los alumnos con las dinámicas establecidas en el ciberespacio?, así como, ¿Qué resultados ha tenido esta educación telemática de 10 meses, y que prospectiva tendrá para el beneficio de los estudiantes que ahora lo están cursando, y, en consecuencia, para los de nuevo ingreso?, por último ¿Cuáles son las características de las TIC's que atraen a los alumnos a tomar una educación a distancia?.

Por supuesto, no podemos dejar de lado lo que ocurre con el virus que ha provocado el colapso mundial de los sistemas de salud, de las economías y que ha dejado miles de muertos, lo que nos lleva a contemplar algunos aspectos que influirán en la reanudación pronta o programada de las actividades presenciales, si el escenario empeora, tendremos que contemplar: ¿Qué puede suceder si ingresan al país otras variantes del virus Covid-19?, ¿Qué imponderables pueden suceder?, ¿Cuáles serán los cisnes negros?, ¿Cuáles serán los comodines ${ }^{1}$ ? ¿Cómo se ha ido desarrollando esta cotidianidad y que podemos esperar?, ¿Cómo se va a resolver?; desde estas preguntas podemos plantear escenarios que mejoran y que empeoran con el pasar de los días, aunque la constante es que las actividades escolares serán las últimas en retomarse, pues corresponden al semáforo verde.

Ahora bien, en términos urbanos, no cabe duda de que la pandemia llegó a modificar la ciudad, aunque no es la primera vez, pues las epidemias fueron parteaguas para generar nuevas trazas e infraestructura que permitieran la circulación de aguas negras, aire, mercancías y personas. Para el presente inmediato y el futuro a corto plazo, podemos apreciar una exacerbación de la dicotomía público-privado, con la frase "quédate en casa" se reafirma que lo privado es el lugar de la salud, la seguridad y el cuidado, mientras que salir a lo público supone riesgo de contagio o un contagio inminente, al estar en contacto con objetos y sujetos extraños, en este caso, incluso lo privado como los centros comerciales es "público" al albergar a multitudes.

La interacción con el medio urbano ha sido profundamente modificada, significada desde el miedo, la precaución y el riesgo constante. Por su parte, la relación con la naturaleza confirma sus bondades, pues el contagio solo puede venir de otra persona, esto desde la concepción de Bergue (1987). Por su parte, las atmósferas, ambientes, memorias, códigos, reglas, ritos y símbolos, que se establecían en las aulas de la ESIA Tecamachalco y Unidad Regina, dejaron de pertenecer a la

1 Imponderables; Es un elemento o hecho que sucede de manera inesperada e inevitable y tiene consecuencias que no se pueden conocer o precisar. Cisnes Negros; este término se entiende como algo sorpresivo, que tiene un gran impacto tras lo sucedido, por lo regular lo relacionamos con hechos históricos (retrospectiva), es decir, una acción como si hubiésemos esperado. Comodines: son los sucesos que tienden a interferir a los resultados asociados a la prospectiva, planificación futura y la investigación, asi mismo, asumen un desarrollo y tendencias. 
dualidad naturaleza-cultura y se trasladaron o migraron al ciber-espacio por necesidad, aun ámbito que esta dinámica telemática no se había utilizado o por lo menos no de manera generalizada, por lo que, todas las interrogantes antes mencionadas, plantean una reflexión interesante que podemos resumir en la siguiente pregunta: ¿Cuáles han sido las ventajas y las desventajas de tomar clases a distancia en la SEPI de la ESIA Tecamachalco?, esto sin perder de vista las condiciones emocionales y las adaptaciones de los entornos domésticos.

Para lo antes mencionado, consideramos al espacio como "un proceso abierto" y dinámico, de circulación en el que las mercancías, las personas, las referencias culturales y las relaciones sociales se han ido globalizando, de manera que las calles y los medios de transporte van conviviendo cada vez más de cerca con los medios de comunicación digitales asociados a estas TIC'S o en algunos casos como es el de la educación, han sido completamente transformados, por lo menos de manera temporal:

La articulación entre tecnología, economía, sociedad y espacio es un proceso abierto, variable e interactivo, parece claro que en la sociedad de la información lo global condiciona lo local y los flujos electrónicos estructuran la economía a partir de relaciones entre unidades espacialmente distantes (Borja y Castells, 1997).

Así, la alternativa a lo presencial son los espacios virtuales, como medio "intangible" de comunicación e interacción, de manera que estamos hablando de ciberespacio, palabra que aparece por primera vez en 1984 "en una novela de William Gibson: Neuromante. Una obra ciberpunk protagonizada por un héroe que quiere volver al mundo online del que fue expulsado" (LINUBE, 2020), sin embargo, existe propiamente desde la aparición del internet, que inició después de la Segunda Guerra Mundial como un espacio tangible que ha llevado alrededor de 50 años para pasar de sus principios bélicos a su incursión en la vida cotidiana en buena parte del mundo, teniendo como sus momentos más importantes el final de la década de los 90's del siglo XX y las dos primeras décadas del siglo XXI.

Se debe agregar que, la interacción entre espacio físico y virtual se da en el momento en que los actos realizados en un punto tienen implicaciones directas en la vida de otras personas, a partir de efectos en la economía, en la cultura y la educación, por mencionar solo algunos aspectos, sin perder de vista que todo está condicionado por la posibilidad de acceso y funcionamiento de dispositivos electrónicos.

Al "interior" de la red o de los espacios virtuales, como cibernautas (alumno que interactúa en el ciberespacio) nos encontramos frente a un usuario que experimenta de manera multisensorial, es decir, que las barreras físicas impuestas por una pantalla (calidad del entorno), provocan una noción cartesiana del espacio.

Además, el impacto generado por dichos medios digitales suscita por lo menos dos vertientes, una a favor y otra en contra, una que ve en los avances tecnológicos y de la información todas las ventajas y beneficios, frente a la que está en contra, planteando un escenario distópico del futuro y romantizado el pasado; los primeros son definidos como e-tópicos "tecnófilos", son quienes disfrutan de los nuevos lugares de carácter electrónico, compran, juegan, conocen personas e incluso visitan lugares geográficamente distantes por medio de sus dispositivos; mientras del otro lado de la moneda encontramos a los distópicos "tecnofobicos", son, como se mencionó, quienes temen por la destrucción de los lugares en el sentido urbano, social y arquitectónico, de manera que sean sustituidos por espacios intangibles que desdibujen las relaciones propiamente humanas. 
La tecnofilia o e-topía entiende que la ciudad es un nuevo lugar de carácter electrónico y la tecnofobia o distopía supone la destrucción de la noción de lugar. Los impulsores de la e-topía ven en la tecnología "un nuevo orden social caracterizado por la democracia, el retorno a la comunidad y a sostenibilidad: democracia en tanto que el ciberespacio se presupone como un medio donde la información estará disponible para todos [...] todos podrán expresarse libremente (García, 2004, p.178).

En E-topia de Mitchell (2000) retomada por García Vázquez (2004) como una más de sus capas urbanas en Ciudad Hojaldre, existe la desmaterialización (desaparición física), desmovilización (todo dentro de un mismo espacio para evitar salir) y por último, el funcionamiento inteligente o autónomo de los edificios (con el uso de la tecnología electrónica y digital), esto con la constante interacción de los habitantes, dando como resultado interacciones multiperceptibles.

Está claro que desde la popularización y masificación del internet, inició un proceso que ha tenido como uno de sus principales objetivos la educación, de modo que los avances tecnológicos en el siglo XXI han sido determinantes para la vida cotidiana estudiantil, debido a las constantes comunicaciones, interacciones simuladas y construcciones virtuales de asesorías o clases, por lo tanto, la educación telemática, se ha ido incorporando paulatinamente, con una irrupción total que podemos fechar desde mediados del mes de marzo de 2020, momento desde el cual los cibernautas (estudiantes) han modificado y adaptado sus atmósferas, con ello, las reglas y rituales cotidianos también se han transformado para formar parte de espacios cerrados y "controlados", como lo menciona Tena (2007), desde su perspectiva urbana, al realizar una homologación de "la forma tecnológica a la forma urbana", en este caso, el ciberespacio se plantea como: "un nuevo espacio generado con la introducción de las nuevas tecnologías de la información en la ciudad tradicional" (Tena, 2007:231), un nuevo espacio que interactúa, en algunos casos sustituye funciones o alterna con la ciudad tradicional, originando nuevas prácticas y formas de comunicación.

Como parte de esas nuevas prácticas que se van haciendo cotidianas, encontramos el tomar clases a distancia, utilizando dispositivos electrónicos como: televisión, celulares, computadoras o tabletas conectadas a una red digital, de manera que, tanto el internet como los dispositivos electrónicos se han convertido en herramientas indispensables, por lo que la compra, tanto de aparatos (hardware) como de aplicaciones (software), se ha incrementado de manera importante durante los últimos meses como lo podemos observar en las siguientes estadísticas en Tabla 2:

Tabla 2: Usuarios de Internet, según principales usos, 2019. Fuente: Procuraduría Federal del Consumidor, 2020.

\begin{tabular}{cc}
\hline Entretenimiento & $91.5 \%$ \\
\hline Obtener información & $90.7 \%$ \\
\hline Comunicarse & $90.6 \%$ \\
\hline Redes sociales & $87.8 \%$ \\
\hline Apoyar educación y capacitación & $83.8 \%$ \\
\hline Acceder a contenido audiovisual & 83.8 \\
\hline Descargar software & $48.0 \%$ \\
\hline Leer periódicos, revistas, libros. & $47.3 \%$ \\
\hline Interactuar Gobierno & $35.6 \%$ \\
\hline Ordenar compras Productos & $22.1 \%$ \\
\hline Utilizar servicios nube & $19.4 \%$ \\
\hline Operaciones banca en línea & $16.8 \%$ \\
\hline Ventas Internet & $19.3 \%$ \\
\hline
\end{tabular}


En la tabla anterior (Tabla 2), se muestran los principales usos del internet, podemos ver que, las variables: "obtener información”, y, "apoyar la educación y capacitación”, se encuentran dentro de los primeros lugares, con lo que deducimos que aún antes de la pandemia ya formaban parte de las actividades cotidianas de los usuarios, pues, realizar tareas en todos los niveles educativos requiere de información que proviene en su mayoría de libros digitalizados, páginas de internet o redes sociales, además de que las entregas de trabajos o avances también se envían por correo electrónico, aunque las actividades presenciales aún no eran sustituidas por la "presencia virtual".

A los pocos días de iniciada la contingencia sanitaria, es decir, a mediados del mes de marzo, la Asociación Mexicana de Ventas Online (AMVO), realizó un estudio que concluyó durante el mes de abril del 2020, dicho análisis muestra el impacto económico que ha tenido la pandemia en el ámbito de compra-venta de software y hardware, con aumentos muy significativos orientados a la resolución de necesidades que anteriormente se atendía de forma presencial como realizar pagos, asistir a eventos o asistir a las escuelas. En la Tabla 3 encontramos el porcentaje de visitantes a sitios web que tienen la intención de adquirir tecnología, ya sea aparatos o paquetería para sus dispositivos:

Tabla 3: promedio de visitantes únicos diarios. Fuente: Asociación Mexicana en venta online (AMVO), 2020.

\begin{tabular}{ccc}
\hline & Computer Software & \\
\hline Semana & Marzo 9 & 2068 \\
11 & Marzo 15 & 2218 \\
Semana & Marzo 16 & $+7 \%$ \\
12 & Marzo 22 & 2875 \\
Semana & Marzo 23 & $+30 \%$ \\
13 & Marzo 29 & 3158 \\
Semana & Marzo 30 & $+10 \%$ \\
\hline 14 & Abril 5 & \\
\hline
\end{tabular}

Promedio de visitantes únicos diarios. Sitios donde los usuarios pueden comprar software de computadoras

\begin{tabular}{ccc}
\hline & Computer Hardware & \\
\hline Semana & Marzo 9 & \multirow{2}{*}{975} \\
11 & Marzo 15 & 973 \\
Semana & Marzo 16 & $0 \%$ \\
12 & Marzo 22 & 1072 \\
Semana & Marzo 23 & $+10 \%$ \\
13 & Marzo 29 & 1056 \\
Semana & Marzo 30 & $-1 \%$ \\
\hline 14 & Abril 5 & \\
\hline
\end{tabular}

Promedio de visitantes únicos diarios. Sitios donde los usuarios pueden comprar computadoras, hardware y accesorios de computadoras.

\begin{tabular}{ccc}
\hline & Electrónicos & \\
\hline Semana & Marzo 9 & 688 \\
11 & Marzo 15 & 650 \\
Semana & Marzo 16 & $-3 \%$ \\
\hline 12 & Marzo 22 & 769 \\
Semana & Marzo 23 & $+18 \%$ \\
\hline 13 & Marzo 29 & 1056 \\
Semana & Marzo 30 & $-1 \%$ \\
\hline 14 & Abril 5 & \\
\hline
\end{tabular}

Promedio de visitantes únicos diarios. Sitios donde los usuarios pueden comprar electrónicos 
En la Figura 1 se muestra específicamente el incremento en las ventas de aparatos electrónicos:

\section{Electrónicos}

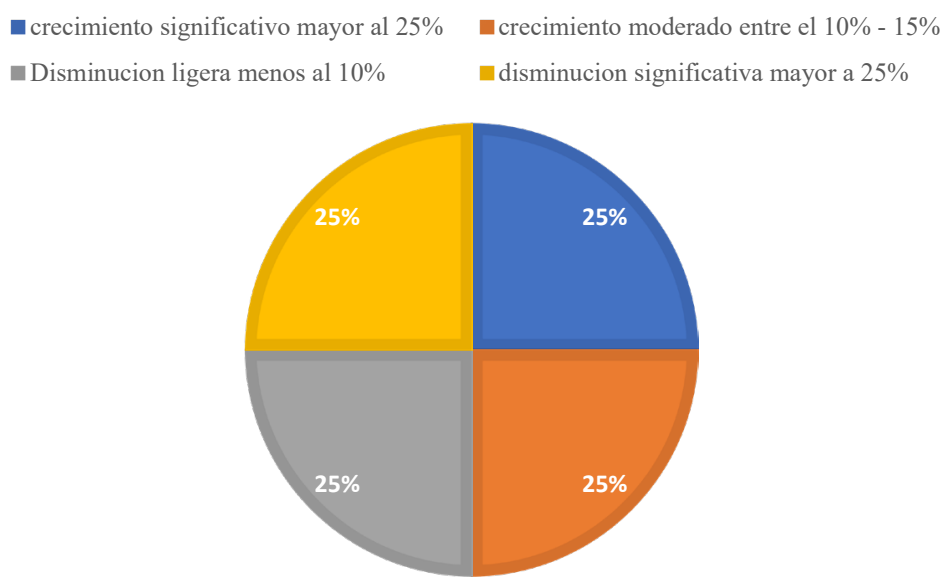

Figura 1: Impacto en el Volumen de Negocios. Fuente: Asociación Mexicana en venta online (AMVO), 2020.

En la siguiente Tabla 4 se muestra el aumento en el uso de aplicaciones o páginas de internet para realizar actividades, debido a que el ciberespacio se ha convertido en el principal medio para evitar la asistencia a espacios que supondrían riesgos de contagio:

Tabla 4: Servicios comprados online durante la pandemia. Fuente: Asociación Mexicana en venta online (AMVO), 2020 .

\begin{tabular}{ccc}
\hline & Primera vez & Últimos siete dias \\
\hline Banca móvil & $5 \%$ & $55 \%$ \\
\hline Telefonía móvil & $5 \%$ & $51 \%$ \\
\hline $\begin{array}{c}\text { Pagos servicios (internet, luz, } \\
\text { agua, gas) }\end{array}$ & $5 \%$ & $47 \%$ \\
\hline Movilidad urbana & $2 \%$ & $31 \%$ \\
\hline Entretenimiento & $5 \%$ & $29 \%$ \\
\hline Educación & $3 \%$ & $21 \%$ \\
\hline Espectáculos eventos & $2 \%$ & $20 \%$ \\
\hline Viajes & $0 \%$ & $18 \%$ \\
\hline
\end{tabular}

Por último, se muestra el resultado de las ventas durante el "Buen Fin"; en la Tabla 5 se presenta el periodo del 2019, y en la Tabla 6 el periodo del 2020, dentro de estas dinámicas de "shopping", los electrónicos y celulares tuvieron un aumento en 2020 comparado con el año anterior, por otro lado, llama la atención que el contenido digital empezó a figurar dentro de las compras, ya que durante 2019 no era contemplado como una "mercancía", aunque ya se encontraba en diversas plataformas de transmisión continua (streaming).

Tabla 5: Top 15 de categorías más compradas; durante el buen fin del 2019. Fuente: Asociación Mexicana en venta online (AMVO), 2020.

\begin{tabular}{cc}
\hline Electrónicos & $31 \%$ \\
\hline Celulares & $22 \%$ \\
\hline Contenido digital & Sin registro \\
\hline
\end{tabular}


Tabla 6: top 15 de categorías más compradas; durante el buen fin 2020. Fuente: Asociación Mexicana en venta online (AMVO), 2020.

\begin{tabular}{cc}
\hline Electrónicos & $34 \%+3 \mathrm{pp}$ \\
\hline Celulares & $23 \%+1 \mathrm{pp}$ \\
\hline Contenido digital & $12 \%$ \\
\hline
\end{tabular}

Por otro lado, encontramos el aspecto legal, que en términos educativos ha integrado lineamientos para que las tecnologías contribuyan al aprendizaje, de manera que la Ley General de la Educación que a continuación se expone en su capítulo II, nos da los elementos para considerar las herramientas digitales como parte integral del sistema educativo:

Emitir los lineamientos generales para el uso responsable y seguro de las tecnologías de la información y comunicación en el sistema educativo. (Ley General de Educacion, 2018, Articulo 12)

Fomentar el uso responsable y seguro de las tecnologías de la información y la comunicación en el sistema educativo, para apoyar el aprendizaje de los estudiantes, ampliar sus competencias para la vida y favorecer su inserción en la sociedad. (Ley General de Educacion, 2018, Articulo 14)

Los aspectos cuantitativos mostrados en las tablas nos permiten reconocer el aspecto comercial de las necesidades que plantea la nueva normalidad, que trajo consigo la digitalización de operaciones bancarias, de servicios, laborales y de manera importante, de actividades escolares, para lo cual se requiere del servicio de internet y por lo menos de un dispositivo electrónico para acceder, es así que buena parte de las compras realizadas en tiendas virtuales responden a la resolución de necesidades asociadas con la comunicación y el acceso al ciberespacio. Por su parte, el aspecto legal tiene una orientación más cualitativa al mencionar aspectos como el uso responsable y seguro de las tecnologías.

Continuando con la reflexión, retomamos a Urrieta (2018), quien considera que el uso de las tecnologías electrónicas y digitales ha tenido un gran impacto en la cotidianidad, de manera que, ya no es posible dejar de usarlas sin generar "una perturbación" en la vida cotidiana, sobre todo de las generaciones que mantienen una relación tan estrecha con los teléfonos celulares o las computadoras, a tal punto que los asimilan como una suerte de "prótesis", es decir, una implementación artificial al cuerpo y la mente para llevar a cabo actividades, lo cual supone un control y dependencia parcial o total que cabe cuestionar: ¿Quién las crea, quien les da sentido, quien las utiliza, como se utilizan y quien se beneficia realmente? (Urrieta,2018:35).

El proceso de asimilación de las nuevas tecnologías no es nuevo, los teléfonos celulares y las computadoras ya son parte de la vida diaria, sin embargo, para este caso, los estudiantes mantenían una relación parcial con los dispositivos al interactuar con profesores y compañeros a partir de correos electrónicos o grupos de whatsapp, entre otras actividades; por lo demás, la asistencia a las instalaciones de Regina o de la ESIA en Tecamachalco constituían la mayor parte de las labores presenciales, de manera que las reflexiones o dudas surgían y se aclaraban en el momento.

Se debe agregar que las dinámicas escolares se conforman a partir de códigos compartidos entre profesores y alumnos que confluyen en un espacio que es el aula, lugar en el que se comunica a partir del habla y de otros lenguajes que pueden ser incluso corporales, situación que se ve modificada o parcialmente borrada en el ciberespacio, de manera que en la nueva normalidad se irán 
configurando nuevos lenguajes como parte de un "proceso de continua producción, actualización y transformación de modelos simbólicos (en su doble acepción de representación y de orientación para la práctica individual y colectiva), en contextos históricamente específicos y socialmente estr ucturados"(Gimenez,2005:75).

Lo anterior nos lleva a la posibilidad de explicar las recodificaciones o adecuaciones en las prácticas llevadas del espacio real al ciberespacio entendiendo que se trata de una hibridación en la que los espacios arquitectónicos son vividos y vistos desde dispositivos electrónicos, se trata de entornos arquitectónicos que se están resignificando sin perder su carácter histórico, como lo concibe Ruskin (1994), desde las memorias que ubican a las edificaciones como objetos culturalmente trascendentales. Resulta imprescindible tener estas consideraciones para plantear los escenarios prospectivos de la educación telemática y sus implicaciones en los alumnos de la SEPI de la ESIA Tecamachalco.

En el siguiente apartado se realiza un diagnóstico de las formas y dinámicas que los alumnos han adoptado y adaptado al tomar clases a distancia, del mismo modo, se determinarán las variables (ponderables, comodines y cisnes negros) para determinar los escenarios prospectivos (tendencial, futurible, utópicos, y pesimista).

\section{Entrevistas}

Las condiciones en las que se lleva a cabo el trabajo escolar desde casa implican modificaciones al espacio, como puede ser adquirir un escritorio o una silla más cómoda, quizá una computadora más adecuada y contratar servicios de internet más rápidos. Para las alumnas y alumnos de la SEPI, ESIA Tecamachalco, el proceso ha tenido diversas implicaciones que reconoceremos en las entrevistas semiestructuradas que se realizaron de manera virtual, a partir de video llamadas, experiencia de trabajo de campo por demás interesante y que forma parte de esta nueva normalidad.

Las entrevistas fueron contestadas por 10 personas; Ana Miriam Roldán Garcés, Karen Pineda Galindo, Carlos Ivan Gomez Calderon, Lina Balderrabano Ayala, Javier Alejandro Moedano Duran, Ulises Paniagua Olivares, Tashai Pineda Garcia, Moises Calderon Gonzáles, Lina Maria Ávila Saldaña y Carlos Iván Gómez Calderón. Todas cursan un posgrado en la SEPI, motivo por el cual han modificado sus dinámicas al tomar clases, presentar avances y realizar trámites, principalmente.

Con el cuestionario aplicado, se buscó conocer la cantidad de tiempo que los alumnos dedican a las actividades escolares en el ciberespacio, además de preguntar su opinión, tanto de las clases como de las actividades burocráticas, de lo que obtuvimos un promedio de 5 horas diarias destinadas a clases, congresos, revisiones, asesorías y a investigar por cuenta propia "4-5 horas, el mismo tiempo que yo tomo de clases, digamos que tomo de clases 5 horas cuando tengo mis clases que son miércoles y jueves, entonces prácticamente de lunes a sábado estoy conectado académicamente, de 4-5 horas digamos que son entre 24-25 horas más o menos a la semana" (J. A. Moedano., comunicación personal, 19 de enero de 2021).

Con respecto a la opinión que tienen de las labores administrativas y escolares, los entrevistados reconocen las condiciones y la dificultad para adaptarse a ellas, con esfuerzos por parte de secretarias, profesores y de ellos mismos, por lo que, se generó una respuesta empática: "yo creo que también en cierta medida es un poco entendible porque pues es algo se puede decir es un poco nuevo, el que todo se lleve a distancia y el que tuvimos que cambiar un poco cierta parte de nuestra rutina drásticamente para llevar a cabo... pues seguir con nuestras actividades" (L. Valderrabano, 
comunicación personal, 19 de enero del 2021)

Además de preguntar por las sensaciones corporales, emocionales y espaciales, ya que el encierro y las actividades frente a una pantalla generan formas distintas de sentir, de pensar y modifican la relación que se tiene con el entorno doméstico:

Los primeros meses de pandemia, aunado a otras cosas que me pasaron el año pasado, tuve un acompañamiento psicológico, por la psicóloga de la ESIA [...] Por ejemplo en Ciudad de México no tenía ni escritorio ni silla, porque hacía todo mi avance en la escuela o en la biblioteca, en mi casa yo llegaba solo a dormir, pues con la pandemia tuve que comprar sí o sí un escritorio y la silla para tener un espacio donde poder trabajar cómoda, porque no podía trabajar solo en la cama porque era mi espacio pues privado, porque yo trabajo todo lo de la tesis en un lugar privado, no me gusta que estén más personas a mi alrededor mientras trabajo (L. M. Ávila, comunicación personal, 18 de enero del 2021)

Si bien, todos han tenido una relación muy estrecha con el ciberespacio, se mantenía como una opción o una alternativa de la que en todo caso se podía prescindir, sin embargo, la nueva normalidad plantea una experiencia sin precedentes:

En el seminario de narrativas urbanas, yo había intentado hacer unas transmisiones en vivo, cuando hacíamos el seminario de cine de ciudad y arquitectura, llevábamos a los invitados y mientras ellos hablaban yo trataba de filmarlos para que se quedara registrado en la página de Facebook, hubo algunas ponencias que se filmaron como nuevos segmentos, entonces creo que ya había un poquito esos avances, pero pues ya con la pandemia fueron necesarios (U. Pangiagua, comunicación personal, 19 de enero del 2021)

Otro de los tópicos que fueron de especial relevancia en las entrevistas fue el que nos aporta diferentes puntos de vista con respecto al futuro en el que las actividades presenciales podrían ceder buena parte de su lugar al ciberespacio o podrían retomarse como antes, reconociendo que ambos formatos presentan ventajas y desventajas, las opiniones coinciden en que el regreso a las actividades presenciales estará marcado por la utilización más habitual del ciberespacio:

Esta modalidad que ahora tenemos yo creo que se va a quedar en muchos aspectos, no sé si en toda la educación pero sí va a ser ya un complemento en el momento que regresemos presencialmente a clases, esto va a ser un complemento a ese tipo de educación y porque además como ya nos familiarizamos [...] probablemente la transmisión de educación sí sea presencial, pero repito, puede ser complementado con esta nueva tecnología, si no es que, pues empezamos a emigrar a esta nueva modalidad (M. Calderón, comunicación personal, 19 de enero del 2021)

Por otro lado, el IPN, en su modelo educativo constructivista, durante la pandemia ha instruido a los académicos, para desarrollar sus cátedras de manera virtual. "Mira, la verdad es que en la licenciatura nos dieron un curso intersemestral, un curso emergente y hubieron varios cursos, yo entre a uno con el licenciado Julio Rojas, no sé ¿si ubicas al maestro Julio?, el maestro Julio ahorita está en la Unidad de Tecnología y Campus Virtual, entonces él nos dio un curso intersemestral y entré con él y además en la página del Politécnico hay una cantidad impresionante de tutoriales que se hicieron en el Poli o que también nos lo compartían de YouTube entonces pues fue estar viendo tutoriales, la asesoría del curso con el profesor Julio Rojas" (M. Roldán, comunicación personal, 19 de enero del 2021) 
Las entrevistas nos muestran una perspectiva que "humaniza" la experiencia de una nueva normalidad, dándole un carácter subjetivo y vivido desde las historias personales que muestran el constante aprendizaje para generar los medios de adaptación a una realidad que se ubica en buena medida en el ciberespacio pero que produce respuestas emocionales, efectos económicos, formas de aprender y habitar que se encuentran en los ámbitos "tradicionales" de vida.

\section{Escenarios hipotéticos}

Los escenarios en los que se encontrarán los alumnos de la SEPI en la ESIA Tecamachalco estarán marcados por una educación telemática que plantea, de inicio, seguir tomando clases a distancia desde sus lugares de residencia, por lo que la casa se mantendrá como el entorno de interacción vía remota, además de tratarse propiamente de actividades híbridas al estar geográfica y virtualmente situadas, dicha situación definirá los temas de investigación y las metodologías, ya que los trabajos de campo y de investigación serán en su mayoría, digitales.

\section{La educación en el Ciberespacio como parte de la vida cotidiana}

Podemos visualizar como un escenario muy probable, que habrá un gran interés por parte de los alumnos de nuevo ingreso en los temas asociados a la habitabilidad, confort, espacial, salud, tecnologías de la información y virtualidad, de manera que se van a contemplar emergencias sanitarias ya no como algo lejano e improbable sino como parte de la cotidianidad, de manera que la arquitectura y el urbanismo desde todas sus vertientes darán virajes que aporten conocimiento para la resolución y previsión de desastres que tendrán que complementarse con los realizados en las áreas de sismos, inundaciones y demás situaciones que ya se contemplaban en los estudios.

Con respecto a los espacios como son bibliotecas y secretarías de estado o áreas administrativas, se tendrán que digitalizar las bases de datos, para que a los alumnos les sea más fácil acceder a la información necesaria para la realización de investigaciones y de trámites.

A su vez, continuando con los escenarios muy probables, los alumnos asistirán a los coloquios nacionales e internacionales por videollamada, como antes se reservaba a los grandes ponentes, lo que redundará en mayor inclusión académica, aunque habrá condiciones para asistir presencialmente a eventos académicos y festivos que se desarrollen en la escuela.

\section{La maravillosa idea del Ciberespacio en la educación a distancia.}

En consecuencia y como escenario utópico, diremos que se podrá acceder a mejor y más información de todo el mundo desde los hogares, así que la vivienda regresará a un punto central para la realización de actividades laborales y académicas con el ciberespacio como el eje de dinamismo y versatilidad en torno al cual la familia se mantendrá reunida.

\section{La deficiencia del Ciberespacio en la educación a distancia}

A manera de escenario pesimista, no podemos dejar de lado que los límites poco claros entre trabajo y tiempo libre acarrean conflictos y un cansancio proveniente de una mayor carga de labores autoimpuestas, de manera que esto traerá consigo trastornos psicológicos y psiquiátricos derivados del uso de dispositivos que saturan de información y de estímulos.

Continuando con la lógica de escenarios negativos, uno de los más preocupantes será la carencia de empleos, ante la poca importancia que tendrán los conocimientos académicos, frente a las actividades realizadas por los dispositivos electrónicos, de manera que se sustituirá a las personas 
por máquinas.

Por otro lado, la topografía del país y la carencia de recursos económicos, dificultará la conectividad de muchas personas, de manera que los estudiantes de dichas regiones aumentarán su rezago educativo, con lo que, en general, la situación del país se complicará frente al mundo, pues no se podrá subsanar la brecha entre sociedades altamente calificadas y habituadas al nuevo contexto con sociedades tecnológicamente analfabetas.

Esto, sin perder de vista los efectos en el medio ambiente, pues, las tecnologías electrónicas producen demasiado calor, por lo que, el calentamiento global continuará, del mismo modo, cuando ocurran apagones, los alumnos se encontrarán completamente incomunicados y desinformados. Por último, el sedentarismo, será uno de los factores que conllevan a la falta de entusiasmo y productividad, sobre todo si no realizan actividades complementarias a su formación.

Para construir un escenario que contemple los puntos de vista de los entrevistados, diremos que es posible reconocer un consenso que ve en el corto plazo una hibridación mayor entre lo presencial y lo virtual, de manera que quienes viven en otros estados de la República puedan tomar clases desde sus lugares de origen, con reuniones esporádicas, mientras las revisiones de comité y los trámites administrativos se mudarán al ciberespacio, además de ser un apoyo en caso de que los profesores se encuentren en otro lugar de manera circunstancial. Lo anterior presenta un escenario alentador en el que la tecnología se adapta a las necesidades y no como en el caso de la pandemia en la que a partir de la necesidad se toman los medios disponibles para resolver problemáticas.

\section{Conclusiones}

Las reflexiones prospectivas mostradas en este trabajo nos muestran una realidad sin precedentes, sin embargo, los posibles futuros son similares a lo que ya se venía suponiendo desde antes, lo que nos permite reconocer en los ciberespacios y en las hibridaciones un trayecto que ya existía y que fue acelerado por la pandemia, se trata de una novedad en tanto se insertó como opción frente a los contagios de COVID-19. Las adecuaciones arquitectónicas y de mobiliario, así como la adquisición de hardware y software, se convirtieron en requisitos esenciales para la realización de labores escolares, que muy probablemente continuarán postpandemia y que se volverán parte de los escenarios domésticos convirtiendo a la vivienda en centro de trabajo, estudio y descanso.

Cabe destacar que las compras y las ventas giran en torno a tecnologías, paradójicamente se adquieren en línea, de manera que, en la mayoría de los casos es necesario contar por lo menos con un dispositivo para adquirir otros más y para adquirir la paquetería necesaria para realizar las actividades escolares, en el caso concreto de los estudiantes del posgrado de la ESIA, el acceso no ha significado un problema, lo que si ha cambiado es la cantidad de tiempo que se pasa en línea, pues hay que sumar a las actividades escolares, el tiempo libre, las compras y las comunicaciones personales, lo que lleva a un cansancio y al deseo de regresar por lo menos de forma parcial a las actividades presenciales.

A partir de la información recabada podemos reconocer un futuro cercano cada vez más híbrido, con la interacción entre el ciberespacio y los espacios urbano-arquitectónicos, en el que los estudiantes de la SEPI se irán familiarizando con la asistencia virtual, los profesores mejorarán sus técnicas de enseñanza y se llegará a un equilibro cada vez más benéfico para la educación y la investigación. Además, los traslados largos que llevaban horas de un punto a otro de la ciudad se verán reducidos, de manera que las horas en transporte público o en automóvil serán aprovechadas para actividades más productivas, mediante una conexión a internet. 


\section{Recomendaciones}

- Este trabajo conlleva al tema de habitabilidad, ergonomía, etc., por lo que se sugiere abordar más en este tema para entender el sistema que se ha ido generando a partir de este año de pandemia.

- Se debe abordar mucho sobre la pedagogía en la arquitectura y urbanismo, ya que, durante mucho tiempo se consideró que estas disciplinas no se podrían enseñar a distancia.

- La percepción del espacio urbano arquitectónico es fundamental, sin embargo, ¿Cómo se abordaría este tema, utilizando herramientas digitales y electrónicas?

- Abordar el concepto de prospectiva urbana y arquitectónica, puesto, que es una manera de entender más a fondo el algoritmo social que se desarrolla en el espacio urbano arquitectónico.

- Dada la complejidad del problema, es preciso estudiarlo mediante el pensamiento complejo, que aborda Edgar Morin y Rolando García.

\section{Referencias}

Asociación Mexicana en venta online (AMVO). (2020). El impacto del Covid-19 en las ventas en línea. 23 de abril del 2020. [en línea] Consultado el 8 de Octubre del 2020. https:// www.amvo.org.mx/wp-content/uploads/2020/04/AMVO_Estudio2.0_ImpactoCOVID19 VP\%C3\%BAblica.pdf

Asociación Mexicana en venta online (AMVO). (2020). El impacto del Covid-19 en las ventas en línea. 23 de abril del 2020. [en línea] Consultado el 8 de Octubre del 2020. https:// www.amvo.org.mx/wp-content/uploads/2020/04/AMVO_Estudio2.0_ImpactoCOVID19 VP\%C3\%BAblica.pdf

Asociación Mexicana en venta online (AMVO). (2020). Reporte de resultados. "El Buen Fin 2020". [en línea] Consultado el 14 de Enero del 2020. https://www.amvo.org.mx/wp-content/ uploads/2020/12/AMVO_Resultados_BuenFin2020_VP\%C3\%BAblica.pdf

Asociación Mexicana en venta online (AMVO). (2020). Reporte de resultados. "El Buen Fin 2019”. [en línea] Consultado el 14 de Enero del 2020. https://www.amvo.org.mx/wp-content/ uploads/2019/12/AMVO_Resultados_ElBuenFin2019_VP\%C3\%BAblica.pdf

Bergue, A. (1987) Écoumène, introduction à l'étude des milieux humains. Paris, Belin.

Borja J. (2000). Ciudad y espacio público en El espacio público, ciudad y ciudadanía.

Borja, J. y Castells, M. (1997). Local y Global, La gestión de las Ciudades en la era de la información. México, Santillana Ediciones Generales, SA de CV.

Doxiadis, C. y Papioannou, J. (1974), The inevitable city of the future. Nueva York: W.W. Norton \& Company.

Gándara, G. y Osorio Vera, F., coord.. (2014). Métodos Prospectivos. Manual para el estudio y la construcción del futuro. Paidos: México.

García Vázquez, C. (2004), La visión tecnológica de la Ciudad en Ciudad Hojaldre. Gustavo Gilli, Barcelona. 
Giménez Montiel, G. (2005). Teoría y análisis de la cultura. Dirección de publicaciones del Instituto Coahuilense de Cultura: México

Giménez Montiel, G. (2007). Estudios sobre cultura y sus identidades sociales.

LINUBE(2020). España. LINUBE.com. https://linube.com/blog/ciberespacio-no-es-internet/.

Mitchell, W. (2000), E-topia: Urban life, Jim-But nos as we know it. Massachusetts Institute of Technology.

Procuraduría Federal del Consumidor (2020). Todo a un clic Compras sin salir de casa; 02 de septiembre del 2020 [en línea] Consultado el 17 de Diciembre del 2020. https://www.gob. $\mathrm{mx} /$ profeco/articulos/todo-a-un-clic-compras-sin-salir-de-casa?idiom=es

Ruskin, J. (1994). Las Siete lámparas de la arquitectura, México, Ediciones Coyoacán.

Secretaria de Educación Pública (SEP) (1993). Ley General de Educación. Articulo 12; V Bis. Ultima actualización 19 de enero de 2018. México.

Secretaria de Educación Pública (SEP) (1993). Ley General de Educación. Articulo 14; X Bis. Ultima actualización 19 de enero de 2018. México.

Nuñez, R. (2007). Ciudad, cultura y urbanización sociocultural. México, D.F., Plaza Valdés, S.A. de C.V.

Toro González, J. (2019). La Educación 4.0. en Conversus. México, Instituto Politécnico Nacional. [En Línea]. https://e4-0.ipn.mx/wp-content/uploads/2019/10/ipn-conversus-137-educacion4-0-dr-toro-1.pdf.

Urrieta García, S. (2018). Espacio Público: de la memoria urbana al proyecto local. Instituto Politécnico Nacional: México. 